\title{
Contact angle as a powerful tool in anisotropic colloid synthesis
}

\author{
Marlous Kamp ${ }^{1,2 *}$, Bart de $\mathrm{Nijs}^{2}$, Jeremy J. Baumberg ${ }^{2}$ and Oren A. Scherman ${ }^{1 *}$ \\ ${ }^{1}$ Melville Laboratory for Polymer Synthesis, Department of Chemistry, University of Cambridge, Lensfield Road, Cambridge, CB2 \\ $1 \mathrm{EW}$, United Kingdom. \\ ${ }^{2}$ NanoPhotonics Centre, Department of Physics, University of Cambridge, J J Thomson Avenue, Cambridge, CB3 OHE, United \\ Kingdom. \\ * to whom correspondence may be addressed:mkamp@cantab.net, oas23@cam.ac.uk
}

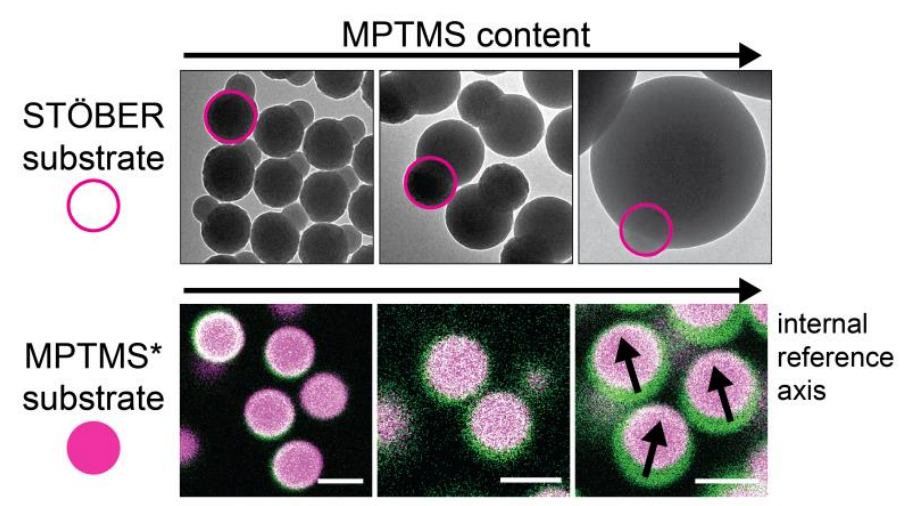

\begin{abstract}
Nucleation and growth is a technique widely used to prepare colloids, in which droplets are adsorbed onto substrate particles. Changing the contact angle of the substrates can greatly alter the morphology of the product particles. Here, we investigate the nucleation and growth of 3-methacryloxypropyltrimethoxysilane (MPTMS) both onto Stöber spheres and onto (cross-linked) MPTMS* spheres. The former results in 'snowman' particles with a cap-shaped MPTMS* compartment, and we show that their morphology is highly controllable via the MPTMS content in the reaction mixture. The contact angle of the MPTMS* compartment decreases with droplet diameter, suggesting that this wetting process is affected not only by surface tension but also by line tension. In contrast to Stöber spheres, MPTMS* substrate particles yield highly reproducible and tuneable 'engulfedsphere' colloids with an internal reference axis (but a homogeneous mass distribution). These engulfed-sphere particles can be fully index-matched for confocal microscopy on account of their homogeneous refractive index. Suitable index-matching mixtures of polar and of low-polar media are presented, where cyclohexyl iodide (CHI) is introduced as a new medium for colloids of high refractive index. Finally, the index-matched engulfed-sphere colloids are self-assembled into (close-packed and long-range) plastic phases, and the particles' rotational diffusion inside the crystal phases is tracked via confocal microscopy.
\end{abstract}

Keywords: nucleation and growth | 3-methacryloxypropyltrimethoxysilane (MPTMS) | Janus colloids | hetero-dimers | rough particles | engulfed-sphere colloids | line tension | wetting | index-matching | rotational diffusion

\section{Introduction}

Wetting is the process of a liquid contacting a surface. Wetting on the nanoscale can be used to produce colloids and nanoparticles of many different shapes. For example, cross-linked polymer particles can be swollen with a monomer; the subsequent expulsion of excess monomer will yield snowman-shaped particles and/or particles with multiple protrusions, as the droplets partially wet the polymer particles.[1-6] Alternatively, droplets can be made to adsorb and grow onto pre-existing substrate particles. An example is the nucleation and growth of 3-methacryloxypropyltrimethoxysilane (MPTMS), as introduced by Sacanna and coworkers.[7,8] Since the substrate colloids used can be of any shape, such a method allows to create a wide range of morphologies.[7-9] Moreover, by exploiting the shape of the substrate particles to induce regiospecific droplet adsorption and growth, it is possible to produce lollipop-shaped colloids[10] and branched colloids[11].

Synthesis mechanisms based on wetting typically involve a surface modification step to set a finite contact angle between the droplet and the parent/substrate particle, as required to obtain particle anisotropy. For example, in the monomer swelling technique in references 1-5, the parent polymer particles are made hydrophilic before monomer expulsion. For MPTMS to nucleate and grow asymmetrically onto silica spheres in ref. 7, the spheres' contact angle is tuned by grafting carbon chains as well as adding a surfactant that absorbs onto the grafted surface.[9] Occasionally, the modification step serves purposes 
additional to tuning the contact angle of the parent/substrate particle. In ref. 6 the stabilizer concentration was adjusted for optimal particle stability during synthesis, while in ref. 5 the parent polymer particle was chlorinated to create product particles with a chemical anisotropy. A point that has remained underexplored, however, is to use different parent/substrate particles with diverse intrinsic (Young's) contact angles, to deliberately change the morphology of the product particles. In the present work, this point is addressed by studying droplet adsorption onto two different types of silica spheres.

The most common and now classic method for silica synthesis was introduced by Werner Stöber in 1968[12] and expanded upon by Bogush,[13] Giesche,[14] Van Blaaderen[15] and others. Such Stöber silica is grown in basic conditions from a silica precursor, typically tetra ethyl orthosilicate (TEOS), and yields amorphous silica spheres of up to $2 \mu \mathrm{m}$ in diameter in a controlled manner.[12,13] Larger particles can be grown by a seeded growth approach,[16] while fluorescent dyes can be incorporated into the seed for studying particle dispersions via confocal microscopy.[17] An alternative way to create silica particles is by hydrolysis and condensation of silane coupling agents ('silanes'), which are silicon compounds with at least one hydrolysable group and one functional group, such as the already mentioned MPTMS. Hydrolysis and condensation of MPTMS is a robust way to create highly monodisperse colloidal spheres.[7,18] The synthesis method consists of two steps: (1) droplet growth and (2) solidification. Under basic conditions, the methoxy groups of the MPTMS hydrolyze, yielding droplets.[19] While siloxane bonds are also formed at this stage, previous studies suggested that these droplets behave more liquid-like than gel-like, as droplets are able to reach energetically favourable locations.[10] After an initiator is added, the methacrylate groups polymerize - or cross-link - and the droplet solidifies. For clarity, we denote such fully condensed and cross-linked MPTMS as: MPTMS*.

Here, we demonstrate that nucleation and growth of MPTMS onto Stöber silica substrates is highly controllable, resulting in morphologies with a cap-shaped MPTMS* compartment. These morphologies are compared to predictions from recent theoretical work on nanoscale wetting, and we show tentative evidence that line tension plays a role in this wetting process. We also show that the Young's contact angle of MPTMS* spheres (as opposed to Stöber silica) facilitates the wetting transition from a cap-shaped state to an engulfing state. Highly reproducible 'engulfed-spheres' are synthesized. This synthesis route for asymmetric engulfed-sphere particles does not require any intermediate grafting step or surfactant adsorption.
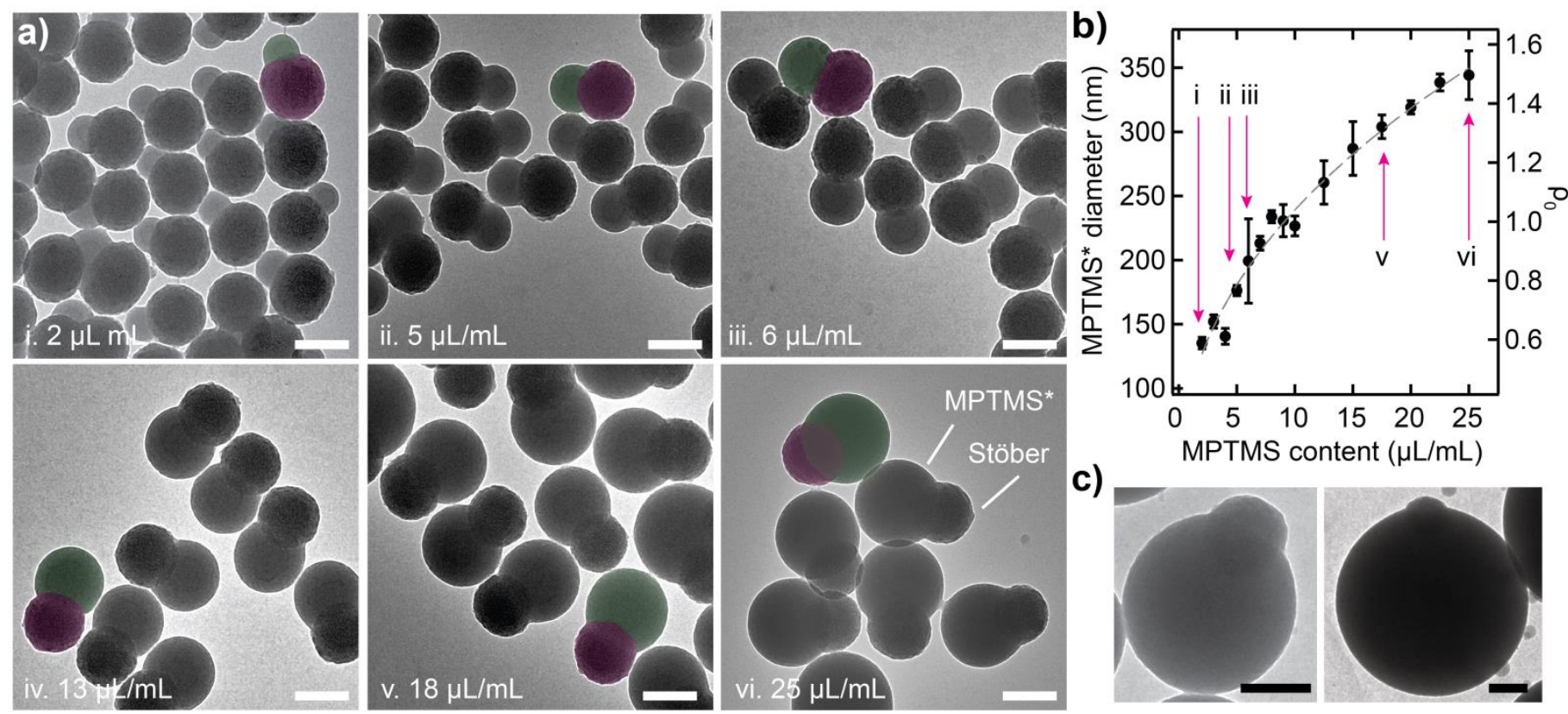

c)

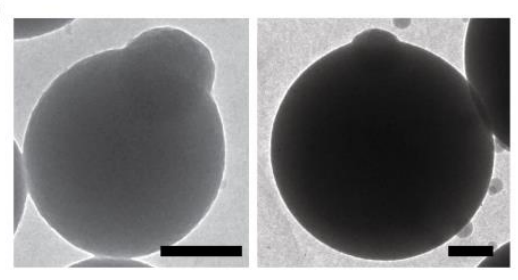

Figure 1 I Hetero-dimers prepared by nucleation and growth of MPTMS onto $230 \mathrm{~nm}$ Stöber silica substrate particles. a) TEM micrographs of hetero-dimers prepared with various MPTMS contents in the reaction mixtures (indicated bottom left). The Stöber silica content was $10 \mathrm{~g} \mathrm{~L}^{-1}$. As a guide to the eye, one Stöber silica substrate particle is labelled in pink and one MPTMS* droplet in green in each image. Scalebars denote $200 \mathrm{~nm}$. b) MPTMS* compartment diameter as a function of MPTMS content in the reaction mixture. The error bars denote size polydispersity, not measurement uncertainty (which is smaller than the symbol size), and is defined as the standard deviation obtained by measuring 10 particles. The dashed line is a fit of the diameter $\mathrm{D}$ to a power law of the added MPTMS content $\mathrm{V}: \mathrm{D}=10\left[2 \pm 5+(8 \pm 4) \mathrm{V}^{0.5 \pm 0.1}\right]$. c) TEM micrographs of a hetero-dimer with the MPTMS* terminating: at the equator of the substrate (left) and at the lower hemisphere of the substrate particle (right). These particles were obtained at MPTMS contents of 33 and $66 \mu \mathrm{L} \mathrm{mL}^{-1}$, respectively. Scalebars denote $200 \mathrm{~nm}$.

An important advantage of these MPTMS*/MPTMS* engulfed-spheres (and other morphologies) is that the particles consist of organosilica throughout. This allows full refractive index-matching for confocal microscopy studies. The particles are indexmatched both in a mixture of polar solvents and in another mixture of low-polar solvents. We introduce cyclohexyl iodide as a 
new medium to index-match colloids of high refractive index such as these MPTMS*/MPTMS* engulfed-spheres. Finally, the engulfed-spheres are self-assembled into (both close-packed and long-ranged) plastic phases. A second important characteristic of the MPTMS*/MPTMS* engulfed-spheres is that they have an internal reference axis without an anisotropic mass distribution, which is of interest for studying rotational motion. As proof of principle, the rotational diffusion of the particles packed into these crystals is monitored via confocal microscopy.

\section{Experimental}

\section{Materials}

Silanes used were 3-methacryloxypropyltrimethoxysilane (MPTMS, 98\%, Sigma Aldrich), aminopropyltriethoxysilane (APTES, 99\%, Sigma Aldrich) and octadecyltrimethoxysilane (OTMOS, 90\%, Acros Organics). The dye derivatives fluorescein isothiocyanate (FITC, $\geq 90 \%$ ) and rhodamine B isothiocyanate (RITC, mixed isomers) were obtained from Sigma Aldrich. Catalysts used were 2,2' azobis(2-methylpropionitrile) (AIBN, 98\%, Aldrich), n-butylamine (99.5\%, Sigma Aldrich), and ammonia solution (35 wt.\%, Fisher Scientific). Pluronic F108 (Mn $\sim 14,600 \mathrm{~g} / \mathrm{mol}$ ) was from Aldrich. Gold nanoparticles (AuNPs, citrate-stabilized, OD1. $80 \mathrm{~nm}$ and $100 \mathrm{~nm}$ ) were purchased from Alfa Aesar. All reactants were used as received. Rhodamine aminostyrene (RAS) was prepared from RITC and aminostyrene (Aldrich, 97\%) according to ref. [20]. The reaction product was dried from ethanol and dissolved in acetone to a concentration of $18 \mathrm{~g} / \mathrm{L}$. PNIPAM-SH (15k) was prepared according to ref. [21].

Water was deionized (resistivity $>18.2 \mathrm{M} \Omega \cdot \mathrm{cm}$ ) using a MilliQ reverse osmosis water purification system (Millipore Corporation). Ethanol (absolute) was from VWR Chemicals. Dispersion media used were 2,2'-thiodiethanol (TDE, 99+\%, Aldrich), cyclohexyl chloride ( $\mathrm{CHC}, \geq 98 \%$, Merck), and cyclohexyl iodide ( $\mathrm{CHI}, 98 \%$, stabilized with copper, Alfa Aesar). CHC was deionized by placing it over aluminium oxide (Sigma Aldrich, type WN-6, activity grade Super I) for $1 \mathrm{~h}$ and over molecular sieves (Acros Organics, $4 \AA$, 8 to 12 mesh) for $6 \mathrm{~h}$.

\section{Methods}

Particles were prepared in a two-step process: (1) nucleation and growth of substrate particles and (2) nucleation and growth of a second MPTMS* compartment onto the substrate particles.

Snowman-shaped Janus (Stöber silica/MPTMS*) particles were prepared as in refs. [7,10]. To this end, Stöber silica particles (average diameter: $230 \pm 1 \mathrm{~nm}$, polydispersity (p.d.): 7\%, Fig. S1) consisting of an FITC-labelled silica core and a non-labelled silica shell were prepared according to refs. $[15,17]$. These particles were functionalized with octadecytrimethoxysilane (OTMOS) by reaction in a mixture of toluene, $\mathrm{n}$-butylamine and OTMOS as in ref. [10] and then dispersed at a volume fraction of $10 \mathrm{~g} \mathrm{~L}^{-1}$ ( $\varphi=$ 0.0045 , corresponding to $7 \cdot 10^{11}$ particles $\mathrm{mL}^{-1}$ ) in an aqueous solution of $0.1 \mathrm{wt} \%$ Pluronic F108. To $3.0 \mathrm{~mL}$ dispersion, $20 \mu \mathrm{L}$ ammonia and a chosen amount (as indicated in text) of MPTMS were added. The reaction mixture was stirred slowly (150 rpm) for $2 \mathrm{~h}$. After adding $6.0 \mu \mathrm{L}$ RAS solution $\left(18 \mathrm{~g} \mathrm{~L}^{-1}\right)$ and $5 \mathrm{mg}$ of AIBN to each, the vials were heated at $70{ }^{\circ} \mathrm{C}$ for $2 \mathrm{~h}$. The product particles were washed and stored in ethanol.

Snowman-shaped Janus (AuNP/MPTMS*) particles were prepared as in ref. [22]. In short, $5.0 \mathrm{~mL}$ of AuNP dispersion (80 nm, citrate-stabilized, optical density $O D=1$, from BBI Solutions) was stirred with $2.0 \mathrm{~mL}$ of $\left[1 \mathrm{~g} \mathrm{~L}^{-1}\right]$ thiolated PNIPAM for $2 \mathrm{~h}$. The AuNPs were then washed three times with water by centrifugation (7 min, $4000 \mathrm{rpm}$ ) and redispersed in $3.0 \cdot 10^{2} \mu \mathrm{L}$ water (particle content $\sim 2 \cdot 10^{11}$ particles $\mathrm{mL}^{-1}$ ). Subsequently, $1.0 \mu \mathrm{L}$ ammonia and a chosen amount of MPTMS were added and the dispersion was stirred for $2 \mathrm{~h}$. Finally, $1 \mathrm{mg}$ of AIBN was added and the reaction completed by stirring at $70{ }^{\circ} \mathrm{C}$ for $2 \mathrm{~h}$. The product particles were washed and stored in ethanol. $100 \mathrm{~nm}$ AuNPs (supplied in $0.1 \mathrm{mg} / \mathrm{ml}$ sodium citrate with stabilizer, OD1) were obtained from Alfa Aesar and their content in the reaction mixture was $7 \cdot 10^{10}$ particles $\mathrm{mL}^{-1}$.

'Engulfed-spheres' (MPTMS*/MPTMS*) were prepared by nucleating and growing MTPMS onto MPTMS* particles. In a typical synthesis of substrate particles, $1.0 \cdot 10^{2} \mathrm{~g} \mathrm{H}_{2} \mathrm{O}$ was mixed with $1.0 \cdot 10^{2} \mu \mathrm{L}$ ammonia solution and a chosen amount of MPTMS (see Fig. S2 for substrate particle size as a function of the added volume of MPTMS). The mixture was stirred vigorously for 1 min, and gently $(300 \mathrm{rpm}$ ) for $2 \mathrm{~h}$. Subsequently, $0.50 \mathrm{~mL}$ RAS solution and $50 \mathrm{mg}$ AIBN were added, and the reaction mixture was stirred at $70^{\circ} \mathrm{C}$ for another $2 \mathrm{~h}$. The resulting MPTMS* spheres were washed three times by centrifugation (20 min. at $4000 \mathrm{rpm}$ ) and redispersed in water to the desired volume fraction for step (2). To $3.0 \mathrm{~mL}$ of the dispersion of substrate particles, $20 \mu \mathrm{L}$ ammonia and a chosen amount (as indicated in text) of MPTMS were added. The mixture was stirred vigorously for 1 min. A fluorescein dye solution was previously prepared by mixing $5.0 \mathrm{mg}$ FITC and $5.0 \mathrm{mg}$ APTES in $3.0 \cdot 10^{2} \mu \mathrm{L}$ ethanol: $0.50 \mathrm{~mL}$ dye mixture was added to the reaction mixtures for every $1 \mathrm{~mL}$ of MPTMS. After stirring gently ( $300 \mathrm{rpm}$ ) for $2 \mathrm{~h}, 5 \mathrm{mg}$ AIBN was 
added and the reaction mixture was heated for $2 \mathrm{~h}$ at $70^{\circ} \mathrm{C}$. The reaction product was washed three times in ethanol by centrifugation (20 min. at $4000 \mathrm{rpm}$ ) to remove unreacted species and secondary particles.

\section{Characterization}

Transmission Electron Microscopy (TEM) was performed on a TECNAI F20 operating at 200 keV (tungsten, LB6). TEM samples were prepared by dropcasting particles onto a formvar/carbon-coated 300 mesh copper grid. Scanning Electron Microscopy (SEM) was performed on a Hitachi S-5500 In-Lens FE SEM operating at 5-15 kV. SEM samples were prepared by dropcasting particles onto a piece of silicon wafer sputter-coated with gold. Contact angles were measured from TEM images using ImageJ $1.50 \mathrm{i}$.

Confocal microscopy was carried out on a Leica SP8 white light inverted confocal microscope, selecting the $495 \mathrm{~nm}$ and $551 \mathrm{~nm}$ line to detect FITC and RAS, respectively, in line sequential scanning mode. Leica's HyD ${ }^{\text {TM }}$ (combined photomultiplier tube and avalanche photodiode) detectors were used for imaging, and Leica LAS AF Lite version 2.6.3 software for analysis. Particle tracking from confocal microscopy images was carried out using a home-written procedure in Igor Pro, version 8.02.

UV-vis spectra were obtained using a home-built set-up with a Micropack DH-2000 halogen lamp and an Ocean Optics QE6500 spectrometer, at room temperature. The integration time was $4 \mathrm{~ms}$. For the absorbance measurements of MPTMS* particles in water/TDE mixtures, a cuvette filled with water was used as background measurement, while for the measurements in $\mathrm{CHC} / \mathrm{CHI}$ mixtures this was a cuvette filled with $\mathrm{CHI}$.
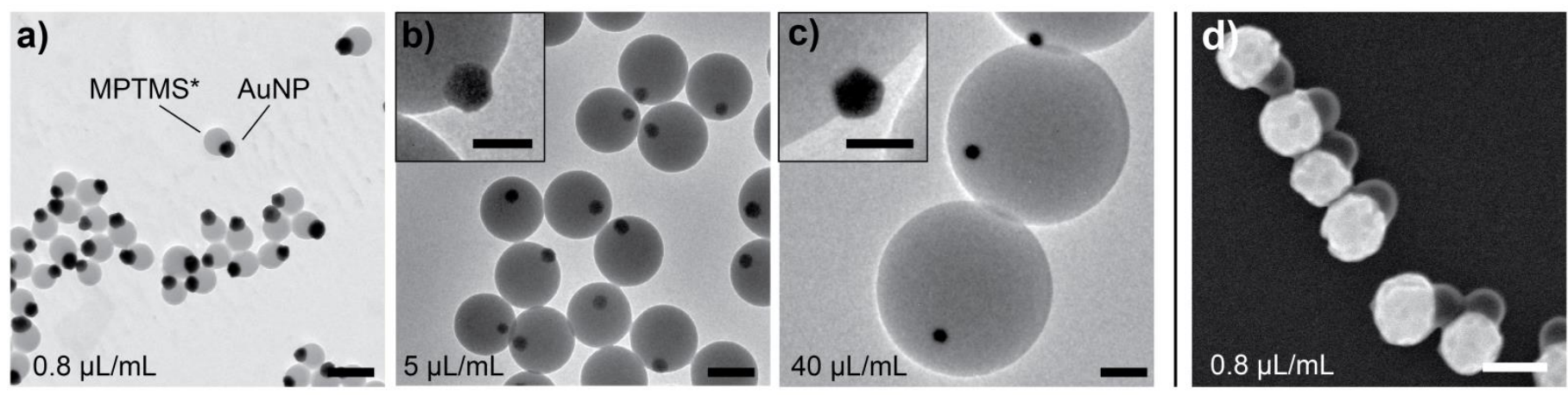

Figure 2 I Hetero-dimers prepared by controlled nucleation and growth of MPTMS onto AuNPs, prepared as described in ref. [22]. a-c) Product particles for $80 \mathrm{~nm}$ AuNP substrates, for increasing MPTMS content (indicated bottom left) in the reaction mixture. Insets display a particle oriented parallel to the electron beam, showing that in these particles the AuNP is only partially engulfed by the MPTMS. d) Product particles for $100 \mathrm{~nm}$ AuNP substrates, at the same MPTMS content in the reaction mixture as in (a). Scalebars denote $250 \mathrm{~nm}$ (a-c) and $100 \mathrm{~nm}$ (insets, d). 
Snowman-shaped Janus colloids can be produced by nucleation and growth of MPTMS onto surface-grafted Stöber silica spheres in the presence of a surfactant.[9,10] We carefully investigated the morphology of these hetero-dimer particles for substrate particles with a diameter of $230 \mathrm{~nm}$ (Fig. S1) for increasing MPTMS content in the reaction mixture (Fig. 1a). In these Transmission Electron Microscopy (TEM) micrographs, the Stöber silica appears darker as it scatters the electron beam more strongly than MPTMS*. The Stöber silica is also distinguishable by its rougher surface compared to MPTMS* (see also Scanning Electron Microscopy (SEM) micrographs in Fig. S3). The hetero-dimers were monodisperse in shape and size up to an MPTMS content of $25 \mu \mathrm{L}$ per $\mathrm{mL}$ reaction mixture. Higher MPTMS contents resulted in large variations in the size of the MPTMS* compartments among particles of the same batch, as well as the formation of spurious MPTMS emulsion droplets (Fig. S4). Below this limit, the volume of the MPTMS* compartment is tuneable to a high precision by the MPTMS content in the reaction mixture (Fig. 1a).

The final MPTMS* compartments' diameter is tuned from $135 \pm 2 \mathrm{~nm}$ (for $2.0 \mu \mathrm{L} \mathrm{mL}^{-1}, p . d .5 \%$ ) to $345 \pm 2 \mathrm{~nm}$ (for $25 \mu \mathrm{LL}$ ${ }^{1}$, p.d.19\%) or in terms of the normalized diameter, from $\rho_{0}=0.59 \pm 0.01$ to $\rho_{0}=1.50 \pm 0.02$ (where $\rho_{0}=R / r$, with $R$ the radius of the MPTMS* compartment, and $r$ the radius of the substrate) (Fig. 1b). The nucleated compartments terminated on the upper hemisphere of the substrate for MPTMS contents below $25 \mu \mathrm{L} \mathrm{mL}^{-1}$, crossing the equator when the diameter reached $\sim 500 \mathrm{~nm}$, or $\rho_{0} \sim 2.2$ (Fig. 1c, left). Particles prepared with $>25 \mu \mathrm{LL}^{-1} \mathrm{MPTMS}$, although polydisperse in shape, showed the MTPMS* compartment terminating at the lower hemisphere of the substrate particle (Fig. 1c:right, and Fig. S4).

Recently, we reported the nucleation and growth of MPTMS onto gold nanoparticles (AuNPs) coated with polymer to prepare hybrid gold/MPTMS* hetero-dimers.[22] These hybrid particles show a similar morphology evolution with MPTMS content as the Stöber/MPTMS* hetero-dimers (see Fig. 2a-c for $80 \mathrm{~nm}$ AuNP substrate particles), demonstrating the generality of the nucleation and growth mechanism for making precisely controlled Janus particles. MPTMS contents up to $40 \mu \mathrm{L}$ per $\mathrm{mL}$ reaction mixture could be used without inducing significant size polydispersity. The MPTMS* compartment diameters observed for such precursor contents were on the order of one micron (Fig.2c, $R=970 \mathrm{~nm}$ ), or $\rho_{0}>12$. The MPTMS* does not engulf the AuNP even for $\rho_{0}=$ 12 (insets in Fig. 2b,c). It was also possible to decrease the diameter of the nucleated compartment down to $67 \pm 1 \mathrm{~nm}$, or $\rho_{0} \sim 0.67$, using larger $100 \mathrm{~nm}$ AuNPs as substrates (Fig. 2d). Due to the facetted shape of the AuNPs, it is difficult to accurately quantify the surface termination of the nucleated compartment, however the embedding of the AuNP into the MPTMS* was not observed to pass $60 \%$ of the AuNP diameter for $\rho_{180}>13$ (Fig. S1b in ref. [22]).

\section{Tuneable MPTMS*/MPTMS* 'engulfed-sphere' particles}

When, instead of Stöber silica spheres, MPTMS* spheres were used as substrate particles, the morphology of the product particles changed significantly. MPTMS* spheres were first grown in a homogeneous nucleation and growth process, in which the sphere diameter was controlled by adjusting the MPTMS content (Fig. S4). The resulting (unmodified) MPTMS* substrate spheres were then subjected to heterogeneous nucleation and growth of MPTMS. Four regimes of morphology were found with increasing amounts of MPTMS in the reaction mixture: I rough particles (Fig. 3a and Fig. S5), II particles with multiple compartments (Fig. 3b), III snowman-shaped particles (Fig. 3c) and IV spherical particles (Fig. 3d-f). Most likely, the surface energy of the MPTMS* substrate allows for multiple droplets to adsorb onto a single substrate, as is the case in the former two morphologies. Rough particles, although not the main focus of this work, are of interest for studies on depletion interaction[3,23] and may have a higher stability in solutions with high ion content.[24] The snowman-shaped particles demonstrate that condensed MPTMS* can accommodate (that is, has a suitable surface energy for) asymmetric nucleation and growth of hydrolysed MPTMS.

Confocal microscopy yielded a qualitative indication of the internal structure of the spherical particles (regime IV), since the MPTMS* substrate was labelled with RAS[10] and the MPTMS* compartment with fluorescein isothiocyanate (FITC)-APTES conjugate[18]. The particles generally consisted of an 'engulfed-sphere' morphology with the FITC-labelled MPTMS* compartment asymmetrically enveloping the RAS-labelled substrate (Fig. 3g-r). The engulfed-sphere morphology was created reliably for MPTMS* substrate particles varying in diameter from $0.44 \mu \mathrm{m}$ (p.d. 7\%, Fig. 3g-j) to $1.78 \mu \mathrm{m}$ (p.d. $3 \%$, Fig. 3o-r). The volume of the nucleated and grown compartment increased with added volume of MPTMS (Fig. 2g-r) and with reduced substrate 
content (Fig. S6). The total particle diameter closely followed the trend for the expected diameter based on added MPTMS volume and substrate content (Fig. S7).
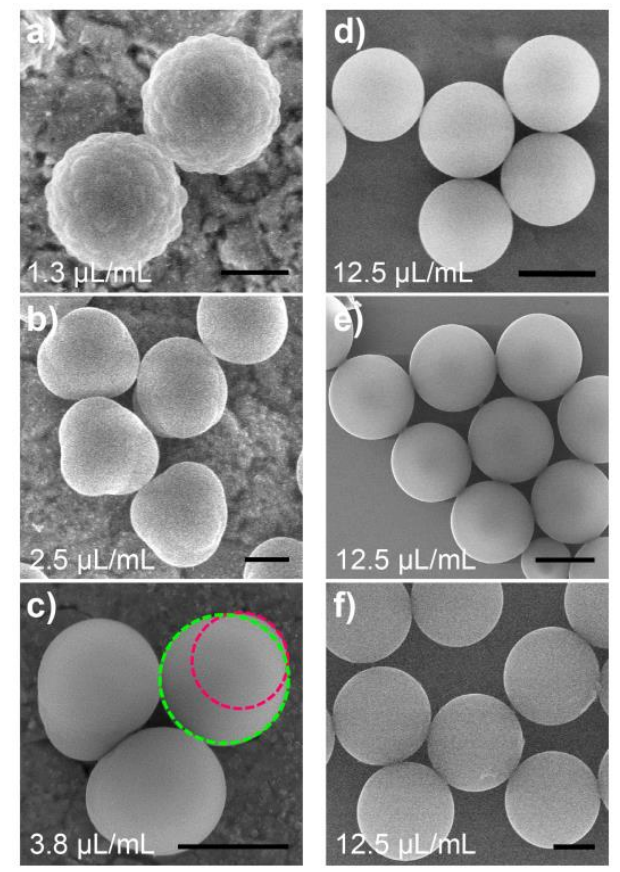

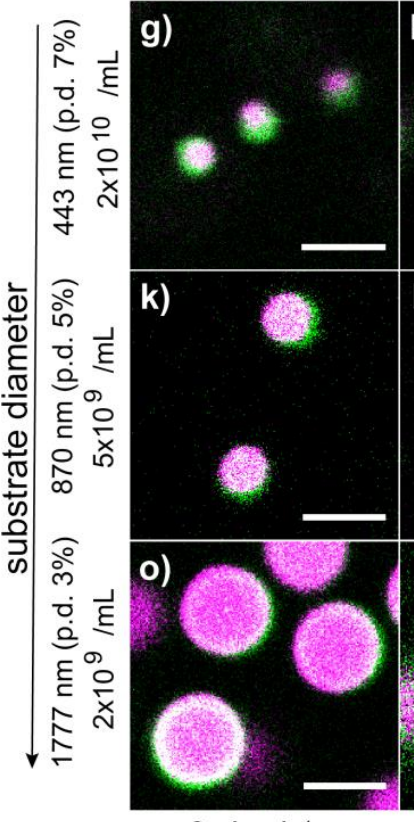

MPTMS
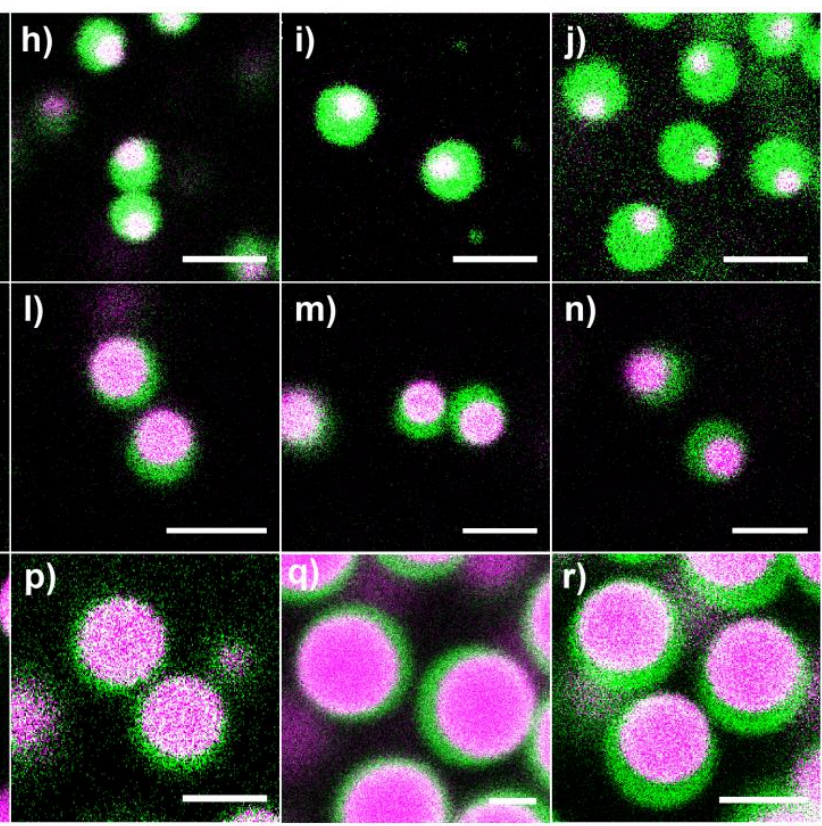

$5 \mu \mathrm{L} \mathrm{mL}^{-1}$

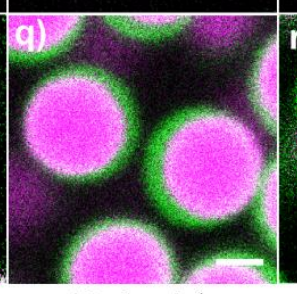

$10 \mu \mathrm{LL}^{-1}$

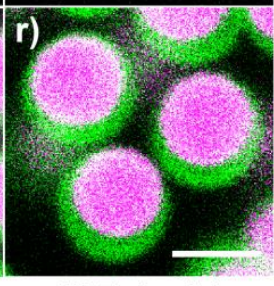

$12.5 \mu \mathrm{L} \mathrm{mL}$

Figure 3 I Nucleation and growth of MPTMS onto MPTMS* spheres. a-c) Shapes of MPTMS*/MPTMS* particles routinely observed for low MPTMS content in the reaction mixture: a) rough particles, $\boldsymbol{b})$ multi-lobe particles and c) snowman-shaped particles, where for one particle the substrate and nucleated compartment are outlined. Scale bars denote $0.5 \mu \mathrm{m}$. $d$-f) SEM micrographs of the engulfed-spheres depicted in $j$ ), $n$ ) and $r$ ), respectively, showing the spherical exterior of the particles. g-r) Confocal micrographs showing the internal structure of the MPTMS*/MPTMS* engulfed-spheres. The substrate particle size, p.d., and substrate content are indicated to the left, and the MPTMS content in the reaction mixture below each column. RAS dye is depicted in pink and FITC dye in green. The dispersion medium is 14 vol.\% water/86 vol.\% TDE. Scale bars denote $2 \mu \mathrm{m}$.

\section{Intermezzo: surface tension and line tension effects in nanoscale synthesis}

The differences in morphology between the Stöber/MPTMS* Janus colloids and the MPTMS*/MPTMS* engulfed-spheres are caused by differences in wetting of the hydrolysed MPTMS onto the substrate particle. Droplet morphology is regulated by minimization of the Helmholtz free energy, which in turn is governed by the surface tension and line tension at the three-phase contact line. Wetting of droplets on (flat) surfaces has been studied theoretically for over half a century, [25] with the influence of line tension recognized soon thereafter.[26-30] Line tension is typically small compared to the surface tensions in macroscopic and mesoscopic systems. For microscopic droplets and substrates, on the other hand, line tension can play a significant role.[26,31-33] In this section, we explore the influence of the contact angle of the substrate and the possible influence of line tension in our syntheses.

For a cap-shaped droplet on a flat surface, line tension introduces a first order correction to Young's equation:[34,35]

$\cos \left(\theta_{E}\right)=\cos \left(\theta_{Y}\right)-\frac{\tau}{\gamma r_{p}}$

where $\gamma$ is the liquid-vapor surface tension and $r_{p}$ the radius of the contact line. In this situation, the line tension can thus be found directly by measuring $\theta_{E}$ as a function of the droplet's contact line, as carried out by Berg et al.[36] and by Heim and Bonaccurso.[34] A simple relation like Eq.(1) does not hold for a droplet on a spherical substrate. The morphology of droplets on a macroscopic spherical substrate (as a function of droplet volume and contact angle) was studied via electrowetting by Eral and coworkers, who found that the partially engulfing state was energetically favourable in all cases. [37] Theoretical studies on the absorption of droplets onto spherical[38-40] and anisotropic substrates[7,10,41,42] have also been performed, but few take line tension into account.[43,44] Recently, Iwamatsu published theoretical work on droplet adsorption on nanoscale spherical substrates, focussing on the influence of line tension.[31,32] In the absence of line tension, droplets take on a cap shape at any droplet volume, keeping a constant contact angle (the Young's contact angle $\theta_{Y}$ ) with the substrate's surface (Fig. 4a, bottom left).[45] When the line tension is not negligible compared to the surface tensions in the system, the equilibrium contact angle $\theta_{E}$ becomes droplet volume dependent and may differ from $\theta_{Y}$ (Fig. 4a, bottom right). Iwamatsu predicts that, in the presence of a 
small positive line tension, the equilibrium contact angle $\theta_{E}$ of a water droplet on a nanoscale spherical hydrophobic substrate decreases monotonically with droplet diameter $\rho_{180}$.[32] Moreover, if the intrinsic Young's contact angle $\theta_{Y}$ is low enough, the cap-shaped droplet constitutes a metastable energy state, with the engulfing droplet morphology having a lower free energy over a wide range of droplet volumes. As a consequence, the droplet morphology can undergo two transitions for increasing droplet volumes: from spherical (i.e. unattached) to cap-shaped, and then from cap-shaped to engulfing (Fig. 4a, top).
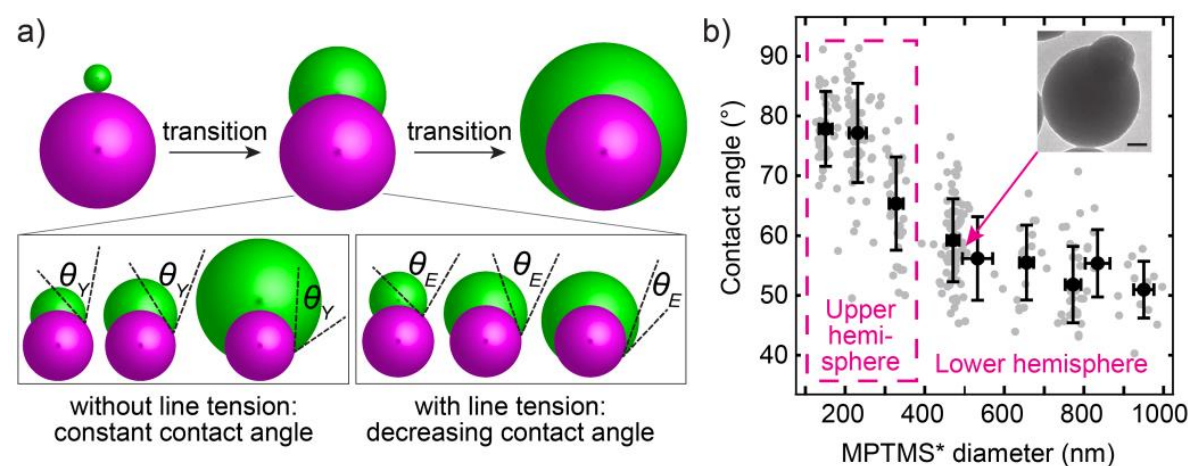

Figure $4 \mid$ a) Schematic representation of the morphologies predicted for wetting of a droplet on a spherical substrate with increasing droplet volume. Figure inspired by ref. [32]. b) Contact angle as a function of MPTMS* compartment diameter for MPTMS*/Stöber hetero-dimer particles. Black markers denote averaged values within the diameter ranges 200-300 nm, 300-400 nm, etc. The error bars denote the standard deviation calculated from all values with the given diameter range. Inset: TEM micrograph of a hetero-dimer with the MPTMS* terminating at the equator of the substrate. Scalebar denotes $100 \mathrm{~nm}$.

When the contact line of an adsorbing droplet passes the equator of a spherical substrate, the equilibrium contact angle $\theta_{E}$ is equal to $\theta_{Y}$.[32] Thus, it is possible to extract an estimate for the Young's contact angle $\theta_{Y}$ [of hydrolysed MPTMS on octadecylgrafted Stöber silica in the presence of Pluronic F108] from the shape of the Stöber/MPTMS* Janus particles, assuming that the cross-linking process does not considerably affect the shape of the MPTMS droplets by volume shrinkage. We measured equilibrium contact angles by drawing the circumferences to the Stöber silica substrate and the MPTMS* compartment, and measuring the angle between the circles' tangents at the crossing points. Only particles placed horizontally on the TEM grid were included, as judged from the horizontal surface termination of MPTMS* (as in e.g. Fig. 1c,left). The equilibrium contact angle decreased monotonically from $78^{\circ} \pm 6^{\circ}$ to $56^{\circ} \pm 8^{\circ}$ with MPTMS* compartment diameter (Fig. $4 \mathrm{~b}$, Fig. S8). Since the contact line passes the substrate's equator for $R \sim 500 \mathrm{~nm}$, an intrinsic Young's contact angle of $\theta_{Y} \sim 60^{\circ} \pm 5^{\circ}$ is estimated. It follows that for MPTMS on MPTMS* substrates, $\theta_{Y}<60^{\circ} \pm 5^{\circ}$, as the droplets transitioned to an engulfing state at similar MPTMS contents. Additionally, the monotonically decreasing contact angle for Stöber/MPTMS* Janus particles indicates that a small positive line tension is present is this system. An estimate for the line tension is found by using the equation[32] $\tau=\tilde{\tau} \sigma_{l v} r$ where $\tilde{\tau}$ is the scaled line tension, $\sigma_{l v}$ is the surface tension of the liquid droplet and $\mathrm{r}$ is the radius of spherical substrate. Assuming a scaled surface tension $\tilde{\tau}=0.1$ as in ref. [32] and a surface tension close to $26 \mathrm{mN} \mathrm{m}^{-1}$ for MPTMS droplets, a line tension of (1.3 - 13).10

${ }^{10} \mathrm{~N}$ is estimated for the presented droplets in the diameter range of $(0.10-1.0) \cdot 10^{3} \mathrm{~nm}$. This estimate is in the same range as other experimental data on heterogeneous nucleation, e.g. ref. [46].

A well-known difficulty in line tension measurements[47] is that the contact line of a droplet can pin on heterogeneities on the substrate,[48] affecting the apparent equilibrium contact angle.[49] Since the Stöber silica particles display surface roughness, as visible in EM images (Fig. S1), contact line pinning cannot be ruled out for these Stöber/MPTMS* Janus particles. In future work, since MPTMS* substrates present a low surface roughness, it could be attempted to graft the MPTMS* spheres with n-octadecyl chains to increase the Young's contact angle to achieve similar morphologies as for the Stöber/MPTMS* Janus particles. However, it is not a trivial task to reach the same surface density of n-octadecyl chains, since the MPTMS* surface contains fewer surface silanol groups than Stöber silica.[50]

\section{Index-matching MPTMS*/MPTMS* engulfed-spheres}

To study self-assembly of particles in detail by confocal microscopy, colloids must be index-matched by a suitable medium. Stöber/MPTMS* hetero-dimers cannot be fully index-matched by any medium, since they consist of two compartments of different refractive index. In contrast, the presented MPTMS*/MPTMS* morphologies consist of MPTMS* throughout, making these colloids of special interest for confocal microscopy. Nevertheless, the refractive index of MPTMS* organosilica is higher than that of most common media[51] and is therefore difficult to match. We introduce two mixtures of solvents which indexmatch MPTMS*; one mixture of polar solvents and one mixture of low-polar solvents. 
The polar solvent 2,2'-thiodiethanol (TDE) is a nontoxic, water-miscible solvent highlighted by Staudt et al.[52] for use in superresolution microscopy. MPTMS* particles were index-matched in aqueous solutions of TDE (Fig. 5a,b). More in detail, the extinction across the $>650 \mathrm{~nm}$ wavelength range (which is used to detect RAS in confocal microscopy) was lowest in $86 \pm 1$ vol. $\%$ TDE (Fig. 5c-d, Fig. S9). This implies that the refractive index of MPTMS* is: $n_{\text {MPTMS }}=1.495 \pm 0.003$, as calculated using the Lorenz-Lorentz equation. MPTMS* particles do experience a small attraction in pure TDE (Video V1), which decreases upon mixing with water.

MPTMS* particles were also successfully index-matched in a mixture of cyclohexyl chloride $\left(\mathrm{CHC}, n_{20}=1.45, \rho=1 \mathrm{~g} / \mathrm{mL}\right.$, $\left.\varepsilon_{20}=7.6\right)$ and cyclohexyl iodide ( $\mathrm{CHI}, n_{20}=1.54$ at $\lambda=656 \mathrm{~nm}, \rho=1.62 \mathrm{~g} / \mathrm{mL}$ )[53], see Fig. $5 \mathrm{e}$. To our knowledge, $\mathrm{CHI}$ has not been used for index-matching before. MPTMS* particles were best index-matched in $49 \pm 1$ vol.\% CHI (Fig. S10). This $\mathrm{CHC} / \mathrm{CHI}$ concentration corresponds to a refractive index $n_{M P T M S^{*}}=1.496 \pm 0.001$, in good agreement with the value found using TDE/water mixtures. Moreover, the $\mathrm{CHC} / \mathrm{CHI}$ mixtures allow to infer the mass density of colloidal MPTMS*. Particles creamed up in $>48 \% \mathrm{CHI}$ (Fig. 4e:bottom, Fig. S10), corresponding to $\rho_{M P T M S^{*}}=1.30 \pm 0.01 \mathrm{~g} \mathrm{~mL}^{-1}$. The mass density of MPTMS* colloids is thus considerably lower than that of conventional Stöber silica $\left(\rho_{\mathrm{SiO}_{2}} \sim 1.9-2.2 \mathrm{~g} \mathrm{~mL}^{-1}\right)[54]$. The MPTMS* refractive index and density found here agree well with recent work by Liu et al,[51] who used a mixture of 45:55 tetralin : trichloroethylene to indexmatch MPTMS*, amounting to a refractive index of $n_{\text {MPTMS }}=1.507$ and density of $\rho_{\text {MPTMS }}=1.24 \mathrm{~g} \mathrm{~mL}^{-1}$.
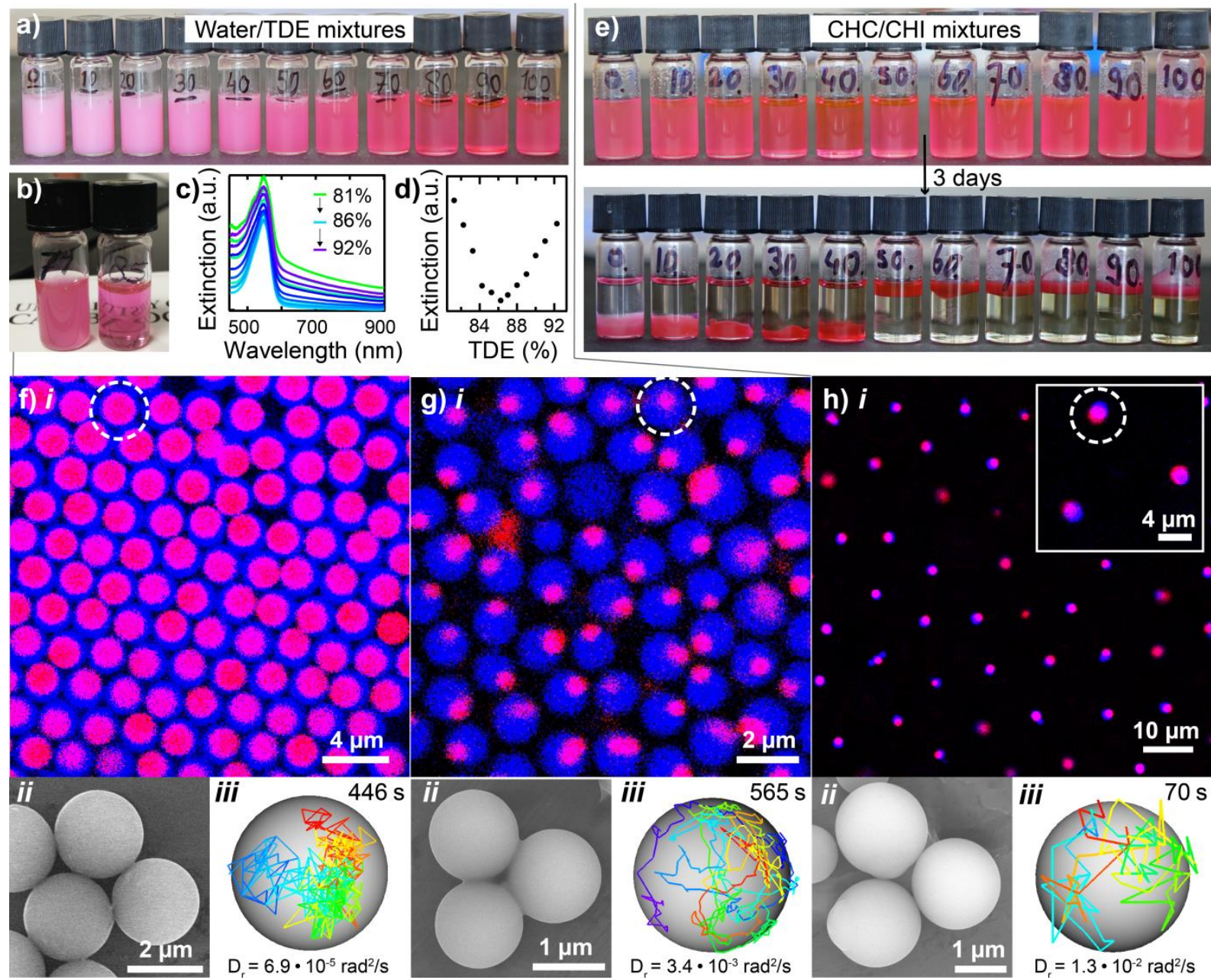

Figure 5 I Index-matching and self-assembly of MPTMS*/MPTMS* engulfed-sphere particles. a) RAS-labelled MPTMS* spheres in mixtures of water and TDE. The MPTMS* content was kept constant at $34 \pm 2 \mu \mathrm{g} \mathrm{mL}^{-1}$. The TDE fraction was increased from 0 vol.\% in steps of 10 vol.\%. b) Photo of the $71 \%$ and $85 \%$ TDE dispersions, displaying the difference in extinction. c,d) UV-vis spectra for a range of dispersions of MPTMS* particles in water/TDE, with TDE fraction increasing in steps of $1 \%$ (c), and absorbance at $650 \mathrm{~nm}$ (d). e) RAS-labelled MPTMS* particles in mixtures of $\mathrm{CHC} / \mathrm{CHI}$. The organosilica concentration was kept constant at $23 \pm 4 \mu \mathrm{g} \mathrm{mL} \mathrm{L}^{-1}$. The $\mathrm{CHI}$ fraction was increased in steps of $10 \mathrm{vol}$.\%. Top: Immediately after preparation. Bottom: After 3 days, showing sedimentation and creaming. f-h) Self-assembly of MPTMS*/MPTMS* engulfed-spheres in short-ranged $(f, g)$ and long-ranged $(h)$ crystals. f:i) Confocal micrograph of MPTMS*/MPTMS* engulfed-spheres prepared from $1.78 \mu \mathrm{m}$ substrate particles in 86:14 TDE:water, close packed by sedimentation. RAS dye (substrates) is depicted in pink and FITC dye (nucleated compartment) in blue. f:ii) SEM image of the particles in f:i. f:iii) Tracked position of the substrate particle relative to the particle's geometrical center, for the colloid circled in f:i. g:i) Confocal micrograph of MPTMS*/MPTMS* engulfed-spheres prepared from 0.44 $\mu$ m substrate particles in 86:14 TDE:water, close packed by sedimentation. g:ii) SEM image of the particles in g:i. g:iii) Tracked position of the 
substrate particle relative to the particle's geometrical center, for the colloid circled in g:i. h:i) Long-ranged crystals of MPTMS*/MPTMS* snowman particles prepared from substrate particles of $0.44 \mu \mathrm{m}$, in CHC at a particle content of $9 \mathrm{~g} \mathrm{~L}^{-1}$. Inset is a zoomed-in micrograph to better show the individual colloids. h:ii) SEM image of the particles in (h:i). h:iii) Tracked position of the substrate sphere relative to the particle's geometrical center, for the colloid circled in (h:i).

\section{Application of MPTMS*/MPTMS* engulfed-spheres: Self-assembly and rotational diffusion}

Colloidal particles explore phase space by translational and rotational diffusion. Understanding the rotational diffusion of spherical particles is of interest, for example since theoretical work shows that it can affect the way in which patchy particles selfassemble into crystals[55] as well as their behaviour in glassy systems/dense fluids[56]. To experimentally study rotational diffusion in-situ requires the individual particles to have a reference axis. Therefore, initial in-situ studies on rotational diffusion used anisotropic particles[57-61] or Janus spheres (i.e. with a chemically modified hemisphere)[62-65]. However, in recent years, several studies have appeared where researchers prepared spherically symmetric particles with an internal anisotropy. These include engulfed-spheres by Liu and Böker,[66] core-shell particles with a number of trace particles containing two differently labelled cores by Schütter et al.,[67] and rough particles where a small number of the particles used to create the roughness is fluorescently labelled by Ilhan et al.[68] Internal anisotropy provides a reference axis with which to track the rotation of spheres, without altering the particles' interaction with the medium. These studies have provided a wealth of new information on the rotational motion of spherical particles, for example that rotational motion reduces by a factor $\sim 2.5$ with volume fraction from freestanding particles to close-packing.[67,69]

The MPTMS*/MPTMS* engulfed-spheres presented here have the same internal structure as the particles pioneered by Liu and Böker,[66] but offer the additional advantage that the particles' center of mass coincides with their geometrical center (as in refs. $[67,68])$. The rotational diffusion is not distorted by any internal mass anisotropy. In this section, we briefly show that the MPTMS*/MPTMS* engulfed-spheres can be assembled into various colloidal crystals and glassy systems, and that their rotational diffusion can be tracked using the internal reference axis.

MPTMS*/MPTMS* engulfed-spheres [prepared from $1.78 \mu \mathrm{m}$ substrate particles, final diameter: $2.36 \pm 0.04 \mu \mathrm{m}$, initial volume fraction: $\phi=0.02$ ] were allowed to sediment in $86 \%$ TDE. The colloids assembled into close-packed structures on account of their spherical shape (Fig. 4f:i). Over 18 crystal layers were recorded, further confirming that the particles are index-matched well in this solvent mixture (Video V2). Smaller MPTMS*/MPTMS* engulfed-spheres [prepared from $373 \mathrm{~nm}(p . d .6 \%)$ substrates, final diameter $1.27 \pm 0.02 \mu \mathrm{m}$, initial volume fraction: $\phi=0.02$ ] also assembled into close-packed, albeit fairly glassy, structures (Fig. 5g:i, and Video V3).

Colloidal crystals with long ranges between particles can be created using media of low ionic strength, e.g. low-polar solvents[70] like CHC and CHI. MPTMS* colloids were not found to be colloidally stable in CHC, even after grafting with octadecyltrimethoxysilane (OTMOS) as in ref. [58]. Most likely, the OTMOS grafting density of MPTMS* is lower than on Stöber silica due to MPTMS* having fewer available silanol groups on the surface.[50] We coated MPTMS*/MPTMS* snowman-shaped particles [1.74 $\pm 0.03 \mu \mathrm{m}$, prepared from $563 \mathrm{~nm}(p . d .2 \%)$ substrates] with a thin $(<10 \mathrm{~nm})$ layer of Stöber silica and then grafted with OTMOS, after which the particles were dispersed in deionized CHC. The colloids formed plastic crystals with inter-particle distances on the order of $10 \mu \mathrm{m}$ (Fig. 5h:i, S11, Video V4). Future research should focus on increasing the grafting density of OTMOS without the need for the thin layer of Stöber silica, so that the particles can form fully index-matched long-range crystals in $\mathrm{CHC} / \mathrm{CHI}$.

The MPTMS*/MPTMS* particles displayed substantial rotational diffusion within both the close-packed and long-range crystals (see Videos V3-V5). Individual particles were tracked by extracting the centers of the substrate particle and the nucleated compartment in the 2D plane. The position of the substrate sphere in 3D with respect to the particle's geometrical center was then calculated using the known particle diameter. This tracking method cannot distinguish between positive and negative zcoordinates, but suffices for small angular displacements. For one particle out of each crystal, the rotational trajectory of the substrate particle on the unit sphere is shown in Figures $4 \mathrm{f}-\mathrm{h}$ :iii. The particles' diffusion coefficients, as calculated from mean square angular displacements (Fig. S12, average of 5 different traces), for each particle are indicated below each reconstruction: they are $\mathrm{D}_{r}=(6.9 \pm 0.1) \cdot 10^{-5} \mathrm{rad}^{2} \mathrm{~s}^{-1}$ for the $2.36 \mu \mathrm{m}$ engulfed-sphere in TDE: water, $\mathrm{D}_{r}=(3.4 \pm 0.1) \cdot 10^{-3} \mathrm{rad}^{2} \mathrm{~s}^{-1}$ for the $1.27 \mu \mathrm{m}$ engulfed-sphere in TDE:water, and $\mathrm{D}_{r}=(1.3 \pm 0.1) \cdot 10^{-2} \mathrm{rad}^{2} \mathrm{~s}^{-1}$ for the $1.74 \mu \mathrm{m}$ snowman-shaped MPTMS*/MPTMS* particle in $\mathrm{CHC}$. Whilst these values are merely indicative as only one particle was analysed for each crystal, the trend is consistent with larger particles displaying slower rotational motion. It would be interesting to investigate to what degree rotational diffusion is 
affected by packing fraction in the long-range crystals of Fig. 5c. Detailed studies of the rotational motion of these particles based on 3D confocal data stacks will be presented in later work.

\section{Conclusions}

Nucleation and growth of MPTMS onto existing structures is a versatile method to create a range of colloidal morphologies. $[7,8,10]$ In this work, we showed that changing the contact angle between the MPTMS and the substrate is a powerful tool to design anisotropic particles, and that the MPTMS content in the reaction mixture controls the particles' dimensions. MPTMS was nucleated and grown both onto (octadecyl-grafted, Pluronic F108-modified) Stöber silica spheres and onto (polymer-coated) AuNPs. A key finding is that the size of the nucleated compartment - and thus the morphology - of the resulting snowman-shaped Stöber/MPTMS* hetero-dimers and AuNP/MPTMS* hetero-dimers can be precisely tuned simply via the precursor content in the reaction mixture.

Pregrown MPTMS* spheres were also used as substrates, here for the first time.[7-10] Several particle morphologies were created for increasing MPTMS content, including rough particles, snowman-shaped particles and engulfed-spheres. The synthesis of these MPTMS*/MPTMS* morphologies does not require any alkane chain grafting or surfactant adsorption, as normally used to tune the surface energy of substrate particles. Via confocal microscopy, we demonstrated that the spherical particles produced at higher MPTMS contents are internally eccentric. This proves that hydrolysed/condensed MPTMS does not completely wet cross-linked MPTMS*.

For microscale droplets, such as the MPTMS droplets in this work, line tension can play a notable role in the wetting process. Theoretical work predicts that, for a small positive line tension, the equilibrium contact angle between a cap-shaped droplet and a spherical substrate decreases with droplet volume.[26,32] A wetting transition to an engulfing droplet is also predicted to occur, provided the Young's contact angle $\theta_{Y}$ is low enough. We measured the contact angle between the MPTMS* compartment and the Stöber silica sphere for the MPTMS*/Stöber hetero-dimers. This contact angle decreased from $78^{\circ} \pm 6^{\circ}$ to $56^{\circ} \pm 8^{\circ}$ as the droplet diameter increased from $135 \mathrm{~nm}$ to $345 \mathrm{~nm}$. Under the assumption that cross-linking and pinning do not affect the shape of the nucleated compartment, this suggests that line tension affects this wetting process. An intrinsic Young's contact angle of $\theta_{Y} \sim 60^{\circ} \pm 5^{\circ}$ was estimated from the contact angle upon passing the substrate's equator. It remains unclear whether a wetting transition is reached for these Stöber/MPTMS* hetero-dimers at large droplet volumes, due to particle size and shape polydispersity observed at high MPTMS contents. In contrast, for MPTMS*/ MTPMS* particles, a wetting transition from a capshaped (in the snowman-shaped particles) to an 'engulfing' droplet for larger droplet size was clearly observed.

The MPTMS*/MPTMS* engulfed-spheres present several attractive properties. These particles (and other presented MPTMS*/MPTMS* morphologies) can be fully index-matched on account of their homogeneous refractive index. Moreover, the particles have an internal reference axis, yet retain a centrosymmetric mass distribution and a chemically homogeneous surface.[51] In addition, they can be facilely prepared uniformly in bulk.[67,68] These aspects make the engulfed-spheres interesting new candidates for studying rotational diffusion by confocal microscopy. The refractive index of MTPMS* is relatively high and requires unconventional dispersion media to achieve full index-matching. The particles were index-matched in mixtures of polar media (86:14 TDE:water) and low-polar media (49:51 CHI:CHC). $\mathrm{CHI}$ is a low-polar medium of high refractive index $\left(\mathrm{n}_{20}=\right.$ 1.54) that has not been used before for index-matching colloids. We found a refractive index of $n_{\text {MPTMS* }}=1.495 \pm 0.003$ and $n_{\text {MPTMS* }}=1.496 \pm 0.001$, respectively, as well as a density of $\rho_{\text {MPTMS* }}=1.30 \pm 0.01 \mathrm{~g} \mathrm{~mL}^{-1}$ which is in agreement with literature[18]. Finally, we prepared close-packed crystals of MPTMS*/MPTMS* engulfed-spheres in 86:14 TDE:water and long-ranged crystals in $\mathrm{CHC}$, tracking the rotational diffusion of the engulfed-spheres inside each of these crystals directly via confocal microscopy.

This work offers various directions for future research. Firstly, the presented Stöber/MPTMS* hetero-dimers of precisely controlled aspect ratio are of interest for self-assembly, allowing to experimentally explore phase diagrams of such snowmenshaped particles[71] in great detail, because of the precisely controlled shape and the ability to label the two compartments with different fluorescent dyes. By further concentrating on the synthesis of MPTMS*/MPTMS* particles close to the transition from snowman-shaped particles to engulfed-spheres, precisely controlled snowman-shaped particles homogeneously composed of MPTMS* may also be created. Secondly, a detailed study into rotational motion of engulfed-spheres in crystals is needed to unveil the exact relation of the diffusion rate with particle size, crystal lattice type and interaction range. Such a study should analyse the 3D position and orientation of the engulfed-spheres, exploiting their internal reference axes. To this end, fast (<1s) image acquisition of 3D confocal data stacks should be employed.[58,68] 
The Cavendish Laboratory Electron Microscopy Suite (R. M. Langford, E. W. Tapley and J. J. Rickard) is thanked for their expert support with electron microscopy. The Gurdon Institute, Cambridge, is thanked for access to confocal microscopy. Dr Vijay K. Rana is gratefully acknowledged for valuable discussions. Funding: MK is grateful to the European Commission for a Marie Curie fellowship (702005, SPARCLEs). BdN acknowledges financial support from the Leverhulme Trust for an Early Career Fellowship with Newton Trust matching funding. This work was also supported by the UK Engineering and Physical Sciences Research Council (EPSRC) [EP/L027151/1] and the Winton Programme for the Physics of Sustainability via a Pump Prime grant.

\section{References}

[1] E.B. Mock, H. De Bruyn, B.S. Hawkett, R.G. Gilbert, C.F. Zukoski, Synthesis of Anisotropic Nanoparticles by Seeded Emulsion Polymerization, Langmuir. 22 (2006) 4037-4043. https://doi.org/10.1021/la060003a.

[2] C. Tang, C. Zhang, J. Liu, X. Qu, J. Li, Z. Yang, Large Scale Synthesis of Janus Submicrometer Sized Colloids by Seeded Emulsion Polymerization, Macromolecules. 43 (2010) 5114-5120. https://doi.org/10.1021/ma100437t.

[3] D.J. Kraft, R. Ni, F. Smallenburg, M. Hermes, K. Yoon, D.A. Weitz, A. van Blaaderen, J. Groenewold, M. Dijkstra, W.K. Kegel, Surface roughness directed self-assembly of patchy particles into colloidal micelles, Proc Natl Acad Sci USA. 109 (2012) 10787. https://doi.org/10.1073/pnas.1116820109.

[4] Y. Li, S. Chen, S. Demirci, S. Qin, Z. Xu, E. Olson, F. Liu, D. Palm, X. Yong, S. Jiang, Morphology evolution of Janus dumbbell nanoparticles in seeded emulsion polymerization, Journal of Colloid and Interface Science. 543 (2019) 34-42. https://doi.org/10.1016/j.jcis.2019.01.109.

[5] B. Peng, H.R. Vutukuri, A. van Blaaderen, A. Imhof, Synthesis of fluorescent monodisperse non-spherical dumbbell-like model colloids, J. Mater. Chem. 22 (2012) 21893-21900. https://doi.org/10.1039/C2JM35229J.

[6] B.G.P. van Ravensteijn, M. Kamp, A. van Blaaderen, W.K. Kegel, General Route toward Chemically Anisotropic Colloids, Chemistry of Materials. 25 (2013) 4348-4353. https://doi.org/10.1021/cm4025606.

[7] S. Sacanna, M. Korpics, K. Rodriguez, L. Colón-Meléndez, S.-H. Kim, D.J. Pine, G.-R. Yi, Shaping colloids for selfassembly, Nature Communications. 4 (2013) 1688. https://doi:10.1038/ncomms2694.

[8] Z. Gong, T. Hueckel, G.-R. Yi, S. Sacanna, Patchy particles made by colloidal fusion, Nature. 550 (2017) 234. https://doi:10.1038/nature23901.

[9] Y. Wang, Y. Wang, X. Zheng, G.-R. Yi, S. Sacanna, D.J. Pine, M. Weck, Three-Dimensional Lock and Key Colloids, J. Am. Chem. Soc. 136 (2014) 6866-6869. https://doi.org/10.1021/ja502699p.

[10] M. Kamp, G. Soligno, F. Hagemans, B. Peng, A. Imhof, R. van Roij, A. van Blaaderen, Regiospecific Nucleation and Growth of Silane Coupling Agent Droplets onto Colloidal Particles, J. Phys. Chem. C. 121 (2017) 19989-19998. https://doi.org/10.1021/acs.jpcc.7b04188.

[11] B. Zhao, D. Li, Y. Long, K. Song, Precisely Endowing Colloidal Particles with Silica Branches, Scientific Reports. 9 (2019) 8591. https://doi.org/10.1038/s41598-019-44742-x.

[12] W. Stöber, A. Fink, E. Bohn, Controlled growth of monodisperse silica spheres in the micron size range, Journal of Colloid and Interface Science. 26 (1968) 62-69. https://doi.org/10.1016/0021-9797(68)90272-5.

[13] G.H. Bogush, M.A. Tracy, C.F. Zukoski, Preparation of monodisperse silica particles: Control of size and mass fraction, Journal of Non-Crystalline Solids. 104 (1988) 95-106. https://doi.org/10.1016/0022-3093(88)90187-1.

[14] H. Giesche, Synthesis of monodispersed silica powders I. Particle properties and reaction kinetics, Journal of the European Ceramic Society. 14 (1994) 189-204. https://doi.org/10.1016/0955-2219(94)90087-6.

[15] A. Van Blaaderen, J. Van Geest, A. Vrij, Monodisperse colloidal silica spheres from tetraalkoxysilanes: Particle formation and growth mechanism, Journal of Colloid and Interface Science. 154 (1992) 481-501. https://doi.org/10.1016/0021-9797(92)90163-G.

[16] H. Giesche, Synthesis of monodispersed silica powders II. Controlled growth reaction and continuous production process, Journal of the European Ceramic Society. 14 (1994) 205-214. https://doi.org/10.1016/09552219(94)90088-4.

[17] A. Van Blaaderen, A. Vrij, Synthesis and characterization of colloidal dispersions of fluorescent, monodisperse silica spheres, Langmuir. 8 (1992) 2921-2931. https://doi.org/10.1021/la00048a013.

[18] C. van der Wel, R.K. Bhan, R.W. Verweij, H.C. Frijters, Z. Gong, A.D. Hollingsworth, S. Sacanna, D.J. Kraft, Preparation of Colloidal Organosilica Spheres through Spontaneous Emulsification, Langmuir. 33 (2017) 81748180. https://doi.org/10.1021/acs.langmuir.7b01398.

[19] C. van der Wel, G.L. van de Stolpe, R.W. Verweij, D.J. Kraft, Micrometer-sized TPM emulsion droplets with surface-mobile binding groups, Journal of Physics: Condensed Matter. 30 (2018) 094005. 
[20] G. Bosma, C. Pathmamanoharan, E.H.A. de Hoog, W.K. Kegel, A. van Blaaderen, H.N.W. Lekkerkerker, Preparation of Monodisperse, Fluorescent PMMA-Latex Colloids by Dispersion Polymerization, Journal of Colloid and Interface Science. 245 (2002) 292-300. https://doi.org/10.1006/jcis.2001.7986.

[21] S.T. Jones, Z. Walsh-Korb, S.J. Barrow, S.L. Henderson, J. del Barrio, O.A. Scherman, The Importance of Excess Poly(N-isopropylacrylamide) for the Aggregation of Poly(N-isopropylacrylamide)-Coated Gold Nanoparticles, ACS Nano. 10 (2016) 3158-3165. https://doi.org/10.1021/acsnano.5b04083.

[22] M. Kamp, B. de Nijs, N. Kongsuwan, M. Saba, R. Chikkaraddy, C.A. Readman, W.M. Deacon, J. Griffiths, S.J. Barrow, O.S. Ojambati, D. Wright, J. Huang, O. Hess, O.A. Scherman, J.J. Baumberg, Cascaded nanooptics to probe microsecond atomic-scale phenomena, PNAS. (2020). https://doi.org/10.1073/pnas.1920091117.

[23] M. Kamp, M. Hermes, C.M. van Kats, D.J. Kraft, W.K. Kegel, M. Dijkstra, A. van Blaaderen, Selective Depletion Interactions in Mixtures of Rough and Smooth Silica Spheres, Langmuir. 32 (2016) 1233-1240. https://doi.org/10.1021/acs.langmuir.5b04001.

[24] Y. Lan, A. Caciagli, G. Guidetti, Z. Yu, J. Liu, V.E. Johansen, M. Kamp, C. Abell, S. Vignolini, O.A. Scherman, E. Eiser, Unexpected stability of aqueous dispersions of raspberry-like colloids, Nature Communications. 9 (2018) 3614. https://doi.org/10.1038/s41467-018-05560-3.

[25] D. Turnbull, Kinetics of Heterogeneous Nucleation, The Journal of Chemical Physics. 18 (1950) 198. https://doi.org/10.1063/1.1747588.

[26] B. Widom, Line Tension and the Shape of a Sessile Drop, Journal of Physical Chemistry. 99 (1995) 2803-2806. https://doi.org/10.1021/j100009a041.

[27] G. Navascués, P. Tarazona, Line tension effects in heterogeneous nucleation theory, The Journal of Chemical Physics. 75 (1981) 2441. https://doi.org/10.1063/1.442309.

[28] R.D. Gretz, Line-Tension Effect in a Surface Energy Model of a Cap-Shaped Condensed Phase, The Journal of Chemical Physics. 45 (1966) 3160. https://doi.org/10.1063/1.1728081.

[29] A.L. Greer, Triple lines in nucleation, Scripta Materialia. 62 (2010) 899-903. https://doi.org/10.1016/j.scriptamat.2010.01.051.

[30] G. Navascuez, L. Mederos, Theoretical analysis of heterogeneous nucleation data: Effects of line tension, Surface Technology. 17 (1982) 79-84. https://doi.org/10.1016/0376-4583(82)90063-2.

[31] M. Iwamatsu, Line-Tension Effects on Heterogeneous Nucleation on a Spherical Substrate and in a Spherical Cavity, Langmuir. 31 (2015) 3861-3868. https://doi.org/10.1021/la504926s.

[32] M. Iwamatsu, Size-dependent contact angle and the wetting and drying transition of a droplet adsorbed onto a spherical substrate: Line-tension effect, Phys. Rev. E. 94 (2016) 042803. https://doi.org/10.1103/PhysRevE.94.042803.

[33] B.M. Law, S.P. McBride, J.Y. Wang, H.S. Wi, G. Paneru, S. Betelu, B. Ushijima, Y. Takata, B. Flanders, F. Bresme, H. Matsubara, T. Takiue, M. Aratono, Line tension and its influence on droplets and particles at surfaces, Progress in Surface Science. 92 (2017) 1-39. https://doi.org/10.1016/j.progsurf.2016.12.002.

[34] L.-O. Heim, E. Bonaccurso, Measurement of Line Tension on Droplets in the Submicrometer Range, Langmuir. 29 (2013) 14147-14153. https://doi.org/10.1021/la402932y.

[35] J.H. Weijs, A. Marchand, B. Andreotti, D. Lohse, J.H. Snoeijer, Origin of line tension for a Lennard-Jones nanodroplet, Physics of Fluids. 23 (2011) 022001. https://doi.org/10.1063/1.3546008.

[36] J.K. Berg, C.M. Weber, H. Riegler, Impact of Negative Line Tension on the Shape of Nanometer-Size Sessile Droplets, Phys. Rev. Lett. 105 (2010) 076103. https://doi.org/10.1103/PhysRevLett.105.076103.

[37] H.B. Eral, G. Manukyan, J.M. Oh, Wetting of a Drop on a Sphere, Langmuir. 27 (2011) 5340-5346. https://doi.org/10.1021/la104628q.

[38] N.H. Fletcher, Size Effect in Heterogeneous Nucleation, The Journal of Chemical Physics. 29 (1958) 572. https://doi.org/10.1063/1.1744540.

[39] M. Qian, Heterogeneous nucleation on potent spherical substrates during solidification, Acta Materialia. 55 (2007) 943-953. https://doi.org/10.1016/j.actamat.2006.09.016.

[40] M. Qian, J. Ma, Heterogeneous nucleation on convex spherical substrate surfaces: A rigorous thermodynamic formulation of Fletcher's classical model and the new perspectives derived, The Journal of Chemical Physics. 130 (2009) 214709. https://doi.org/10.1063/1.3146810.

[41] S.A. Reavley, A.L. Greer, Athermal heterogeneous nucleation of freezing: numerical modelling for polygonal and polyhedral substrates, Philosophical Magazine. 88 (2008) 561-579.

https://doi.org/10.1080/14786430801898636. 
[42] B. Peng, G. Soligno, M. Kamp, B. de Nijs, J. de Graaf, M. Dijkstra, R. van Roij, A. van Blaaderen, A. Imhof, Sitespecific growth of polymers on silica rods, Soft Matter. 10 (2014) 9644-9650.

https://doi.org/10.1039/C4SM01989J.

[43] A. Scheludko, Condensation of vapors on spherical nuclei and the line tension effect, Journal of Colloid and Interface Science. 104 (1985) 471-476. https://doi.org/10.1016/0021-9797(85)90054-2.

[44] K. Padilla, V. Talanquer, Heterogeneous nucleation on aerosol particles, The Journal of Chemical Physics. 114 (2001) 1319. https://doi.org/10.1063/1.1332995.

[45] S. Tao, W. Jiadao, C. Darong, Spreading of a fluid phase on a spherical surface, Journal of Colloid and Interface Science. 358 (2011) 284-289. https://doi.org/10.1016/j.jcis.2011.02.060.

[46] A.I. Hienola, P.M. Winkler, P.E. Wagner, H. Vehkamäki, A. Lauri, I. Napari, M. Kulmala, Estimation of line tension and contact angle from heterogeneous nucleation experimental data, The Journal of Chemical Physics. 126 (2007) 094705. https://doi.org/10.1063/1.2565769.

[47] J. Drelich, J.D. Miller, The line/pseudo-line tension in three-phase systems, Particulate Science and Technology. 10 (1992) 1-20. https://doi.org/10.1080/02726359208906593.

[48] D. Bonn, J. Eggers, J. Indekeu, J. Meunier, E. Rolley, Wetting and spreading, Rev. Mod. Phys. 81 (2009) $739-805$. https://doi.org/10.1103/RevModPhys.81.739.

[49] H. Zhang, S. Chen, Z. Guo, Y. Liu, F. Bresme, X. Zhang, Contact Line Pinning Effects Influence Determination of the Line Tension of Droplets Adsorbed on Substrates, The Journal of Physical Chemistry C. 122 (2018) 1718417189. https://doi.org/10.1021/acs.jpcc.8b03588.

[50] D.N. Mangos, T. Nakanishi, D.A. Lewis, A simple method for the quantification of molecular decorations on silica particles, Science and Technology of Advanced Materials. 15 (2014) 015002. https://doi.org/10.1088/14686996/15/1/015002.

[51] Y. Liu, T. Yanagishima, A. Curran, K.V. Edmond, S. Sacanna, R.P.A. Dullens, Colloidal organosilica spheres for three-dimensional confocal microscopy, Langmuir. 35 (2019) 7962-7969. https://doi.org/10.1021/acs.langmuir.9b00963.

[52] T. Staudt, M.C. Lang, R. Medda, J. Engelhardt, S.W. Hell, 2,2'-Thiodiethanol: A new water soluble mounting medium for high resolution optical microscopy, Microscopy Research and Technique. 70 (2007) 1-9. https://doi.org/10.1002/jemt.20396.

[53] G.H. Jeffery, A.I. Vogel, 364. Physical properties and chemical constitution. Part XVIII. Three-membered and four-membered carbon rings, J. Chem. Soc. (1948) 1804-1809. https://doi.org/10.1039/JR9480001804.

[54] S.R. Parnell, A.L. Washington, A.J. Parnell, A. Walsh, R.M. Dalgliesh, F. Li, W.A. Hamilton, S. Prevost, J.P.A. Fairclough, R. Pynn, Porosity of silica Stöber particles determined by spin-echo small angle neutron scattering, Soft Matter. 12 (2016) 4709-4714. https://doi.org/10.1039/C5SM02772A.

[55] A.C. Newton, J. Groenewold, W.K. Kegel, P.G. Bolhuis, Rotational diffusion affects the dynamical self-assembly pathways of patchy particles, PNAS. 112 (2015) 15308-15313. https://doi.org/10.1073/pnas.1513210112.

[56] S. Marín-Aguilar, H.H. Wensink, G. Foffi, F. Smallenburg, Rotational and translational dynamics in dense fluids of patchy particles, The Journal of Chemical Physics. 152 (2020) 084501. https://doi.org/10.1063/1.5143221.

[57] K.V. Edmond, M.T. Elsesser, G.L. Hunter, D.J. Pine, E.R. Weeks, Decoupling of rotational and translational diffusion in supercooled colloidal fluids, PNAS. 109 (2012) 17891-17896. https://doi.org/10.1073/pnas.1203328109.

[58] B. Liu, T.H. Besseling, M. Hermes, A.F. Demirörs, A. Imhof, A. van Blaaderen, Switching plastic crystals of colloidal rods with electric fields, Nature Communications. 5 (2014) 3092.

[59] T.H. Besseling, M. Hermes, A. Kuijk, B. de Nijs, T.-S. Deng, M. Dijkstra, A. Imhof, A. van Blaaderen, Determination of the positions and orientations of concentrated rod-like colloids from 3D microscopy data, J. Phys.: Condens. Matter. 27 (2015) 194109. https://doi.org/10.1088/0953-8984/27/19/194109.

[60] A. Pal, J.-M. Meijer, J.R. Wolters, W.K. Kegel, A.V. Petukhov, Structure and stacking order in crystals of asymmetric dumbbell-like colloids, Journal of Applied Crystallography. 48 (2015) 238-243. https://doi.org/10.1107/S1600576714028222.

[61] S. Vivek, E.R. Weeks, Decoupling of translational and rotational diffusion in quasi-2D colloidal fluids, The Journal of Chemical Physics. 147 (2017) 134502. https://doi.org/10.1063/1.4996733.

[62] M. Kim, S.M. Anthony, S.C. Bae, S. Granick, Colloidal rotation near the colloidal glass transition, The Journal of Chemical Physics. 135 (2011) 054905. https://doi.org/10.1063/1.3623489.

[63] A. Wittmeier, A.L. Holterhoff, J. Johnson, J.G. Gibbs, Rotational Analysis of Spherical, Optically Anisotropic Janus Particles by Dynamic Microscopy, Langmuir. 31 (2015) 10402-10410.

https://doi.org/10.1021/acs.langmuir.5b02864. 
[64] L.C. Hsiao, I. Saha-Dalal, R.G. Larson, M.J. Solomon, Translational and rotational dynamics in dense suspensions of smooth and rough colloids, Soft Matter. 13 (2017) 9229-9236. https://doi.org/10.1039/C7SM02115A.

[65] X. Wang, M. In, C. Blanc, A. Würger, M. Nobili, A. Stocco, Janus Colloids Actively Rotating on the Surface of Water, Langmuir. 33 (2017) 13766-13773. https://doi.org/10.1021/acs.langmuir.7b02353.

[66] B. Liu, A. Böker, Measuring rotational diffusion of colloidal spheres with confocal microscopy, Soft Matter. 12 (2016) 6033-6037. https://doi.org/10.1039/C6SM01082B.

[67] S. Schütter, J. Roller, A. Kick, J.-M. Meijer, A. Zumbusch, Real-space imaging of translational and rotational dynamics of hard spheres from the fluid to the crystal, Soft Matter. 13 (2017) 8240-8249. https://doi.org/10.1039/C7SM01400G.

[68] B. Ilhan, J.J. Schoppink, F. Mugele, M.H.G. Duits, Spherical probes for simultaneous measurement of rotational and translational diffusion in 3 dimensions, Journal of Colloid and Interface Science. 576 (2020) 322-329. https://doi.org/10.1016/j.jcis.2020.05.026.

[69] M.P. Lettinga, C.M. van Kats, A.P. Philipse, Rotational Diffusion of Tracer Spheres in Packings and Dispersions of Colloidal Spheres Studied with Time-Resolved Phosphorescence Anisotropy, Langmuir. 16 (2000) 6166-6172. https://doi.org/10.1021/la991603v.

[70] A. Yethiraj, A. van Blaaderen, A colloidal model system with an interaction tunable from hard sphere to soft and dipolar, Nature. 421 (2003) 513.

[71] K. Milinković, M. Dennison, M. Dijkstra, Phase diagram of hard asymmetric dumbbell particles, Phys. Rev. E. 87 (2013) 032128. https://doi.org/10.1103/PhysRevE.87.032128. 